\title{
Phase-space interference in extensive and nonextensive quantum heat engines
}

\author{
Hardal, Ali Ümit Cemal; Paternostro, Mauro; Mustecaplioglu, Ozgur E.
}

Published in:

Physical Review E

Link to article, DOI:

10.1103/PhysRevE.97.042127

Publication date:

2018

Document Version

Publisher's PDF, also known as Version of record

Link back to DTU Orbit

Citation $(A P A)$ :

Hardal, A. Ü. C., Paternostro, M., \& Mustecaplioglu, O. E. (2018). Phase-space interference in extensive and nonextensive quantum heat engines. Physical Review E, 97(4), [042127].

https://doi.org/10.1103/PhysRevE.97.042127

\section{General rights}

Copyright and moral rights for the publications made accessible in the public portal are retained by the authors and/or other copyright owners and it is a condition of accessing publications that users recognise and abide by the legal requirements associated with these rights.

- Users may download and print one copy of any publication from the public portal for the purpose of private study or research.

- You may not further distribute the material or use it for any profit-making activity or commercial gain

- You may freely distribute the URL identifying the publication in the public portal 


\title{
Phase-space interference in extensive and nonextensive quantum heat engines
}

\author{
Ali Ü. C. Hardal, ${ }^{1}$ Mauro Paternostro, ${ }^{2}$ and Özgür E. Müstecaplıoğlu ${ }^{3}, *$ \\ ${ }^{1}$ Department of Photonics Engineering, Technical University of Denmark, Ørsteds Plads 343, DK-2800 Kgs. Lyngby, Denmark \\ ${ }^{2}$ Centre for Theoretical Atomic, Molecular and Optical Physics, School of Mathematics and Physics, Queen's University, \\ Belfast BT7 1NN, United Kingdom \\ ${ }^{3}$ Department of Physics, Koç University, 34450 Sartyer, Istanbul, Turkey
}

(Received 27 November 2017; published 18 April 2018)

\begin{abstract}
Quantum interference is at the heart of what sets the quantum and classical worlds apart. We demonstrate that quantum interference effects involving a many-body working medium is responsible for genuinely nonclassical features in the performance of a quantum heat engine. The features with which quantum interference manifests itself in the work output of the engine depends strongly on the extensive nature of the working medium. While identifying the class of work substances that optimize the performance of the engine, our results shed light on the optimal size of such media of quantum workers to maximize the work output and efficiency of quantum energy machines.
\end{abstract}

DOI: 10.1103/PhysRevE.97.042127

\section{INTRODUCTION}

Quantum interference is responsible for fundamental differences between quantum and classical dynamics [1-5]. However, as might be naively believed, the effect of interference is not always to provide advantages and it can also work "against quantumness." According to the path integral formulation of quantum mechanics, constructive interference of quantum paths makes them converge to the classical trajectory in the limit of $\hbar \rightarrow 0$ [6]. It is thus necessary to determine proper parameter regimes for a given system such that the occurrence of interference can be taken as an unambiguous signature of the quantum character of the system.

In this paper, we consider a system of $N$ spin- $1 / 2$ particles interacting through either extensive or nonextensive versions of the anisotropic Lipkin-Meshkov-Glick (LMG) model [7], which is central to many physical problems [8-13]. In the extensive version of such model, the mean energy per spin is finite, while in the nonextensive one it becomes infinite [14]. We use such formulations of the Hamiltonian of our $N$-spin system to describe the working fluid of a quantum heat engine $[15,16]$, assessing the effects that (non-)extensiveness has on the capability of the engine to produce work.

The motivation for such a study stems from a fundamental question: "Can we find genuine interference-induced quantum advantages, scaling up with the number of quantum workers, in the energetics of quantum many-body systems"? The answer to this question, which is central to emerging fields such as quantum biology [17,18] and quantum thermodynamics [19-21], can have practical relevance for the optimization of the performance of quantum heat engines and bioenergetic systems such as artificial light harvesting complexes [22].

We show that fundamental interference-related differences in the performance of engines result from the use of extensive

\footnotetext{
*omustecap@ku.edu.tr
}

and nonextensive work media. Moreover, we illustrate the possibility to achieve significant advantages using certain parameter regimes of nonlinear interactions, in nonextensive work substances. In particular, we find that when the spins in a work medium interact, this can lead to nonmonotonic dependence of the returned work on the number of (working) spins. The effect stems from quantum interference, which can be geometrically and semiclassically explained in the spin phase space [23-25]. Specifically, we consider a quantum Otto cycle [15] and find out that interference is manifested in the work output of the engine $(W)$ through oscillations that depend on the parity of the number of spins in the work medium. We determine the parameter regimes and approach to discern the contribution to such oscillations that can be uniquely associated with phase-space interference.

Moreover, we examine the efficiency and the dependence on $N$ of the marginal productivity $(W / N)$, which both help to further compare extensive and nonextensive quantum heat engines (QHEs). The behavior of the marginal productivity of a quantum work substance can be compared to the "law of diminishing returns" in microeconomics [26]. We identify points of maximum and negative productivity, which can be used to determine the optimal size of the work substance in both the extensive and the nonextensive case. From a practical point of view, such results can be significant for the identification of a cost-efficient scaling law of the productivity of QHEs, complementing what has been achieved using engineered quantum heat baths [27,28]. From the fundamental perspective, instead, phase-space interference can be used to witness the quantum character of a QHE, thus contributing to the current open investigation, in this respect, in the field of quantum thermodynamics [29].

This paper is organized as follows. In Sec. II we introduce generic extensive and nonextensive LMG-type models that we consider as our working substances for a quantum Otto engine. A perturbative method to calculate the work output of the engine is described as well. In Sec. III an introductory 
description of the phase-space interference method for semiclassical geometrical interpretation of quantum interferences is presented. Quantum interference and parity oscillations in the work output of the engine is discussed in Sec. IV using exact and perturbative approaches, which also sets the regime of validity of the perturbative interpretation. The manifestation and isolation of the quantum interference induced oscillations relative to spectral difference induced oscillations in the work output is revealed in Sec. V. We conclude in Sec. VI.

\section{EXTENSIVE AND NONEXTENSIVE QUANTUM OTTO ENGINE}

\section{A. Model Hamiltonians}

We consider two different (nonextensive and extensive) descriptions of the working substance of a quantum Otto engine by using an anisotropic LMG-type Hamiltonian for a set of $N$ (pseudo)spin-1/2 particles, in the absence of any longitudinal magnetic field. The model reads (we choose units such that $\hbar=$ $k_{B}=1$ throughout the paper, with $k_{B}$ the Boltzmann constant)

$$
\hat{H}_{\mathrm{LMG}}=\gamma_{x} \hat{S}_{x}^{2}+\gamma_{y} \hat{S}_{y}^{2},
$$

where we have introduced the collective spin operators

$$
\hat{S}_{\alpha}=\frac{1}{2} \sum_{i=1}^{N} \hat{\sigma}_{\alpha} \quad(\alpha=x, y, z) .
$$

Equation (1) can describe both the extensive and the nonextensive version of the Hamiltonian model, the latter being retrieved by performing the scaling $\gamma_{x, y} \rightarrow \gamma_{x, y} / N$ of the coefficients.

Simulations of the LMG model have been proposed based on a Bose-Einstein condensate trapped in a double-well potential [11], circuit quantum electrodynamics [30], singlemolecule magnets [31], and toroidal condensates [32]. The model has rotational and spin-flip symmetries. For simplicity, we will limit our study to the subspace of symmetric Dicke states corresponding to the maximum total spin sector with $S=N / 2$. In such a manifold, we look for the differences between extensive and nonextensive models in the behavior of the mean energies against the particle number. While, in general, nonextensive models give infinite mean energy per spin in the thermodynamical limit (i.e., for $N \rightarrow \infty$ ), extensive ones lead to finite mean energy per spin in such a limit. According to the so-called Kac rescaling [33], extensivity is recovered at the cost of losing the additivity of the energy, so that the sum of the energies of the subsystems can be different from the total mean energy of the system [14]. While in order to study the phenomenology of nonextensive systems, special forms of entropy have been proposed [34], here we rely on a thermodynamic approach based on the performance of a QHE.

We restrict the analysis to the antiferromagnetic model and thus assume $\gamma_{x, y}>0$. The choice of no external magnetic field makes the model in Eq. (1) noncritical. Phase transitions can lead to nonextensive behavior in heat engines irrespective of their classical or quantum character, which can be used to operate an engine at Carnot efficiency with finite power [35]. Our purpose here is to use quantum interference to reveal quantum signatures of the working substance with extensive and nonextensive descriptions. Recently, quantum and classical interference have been studied in the ferromagnetic LMG

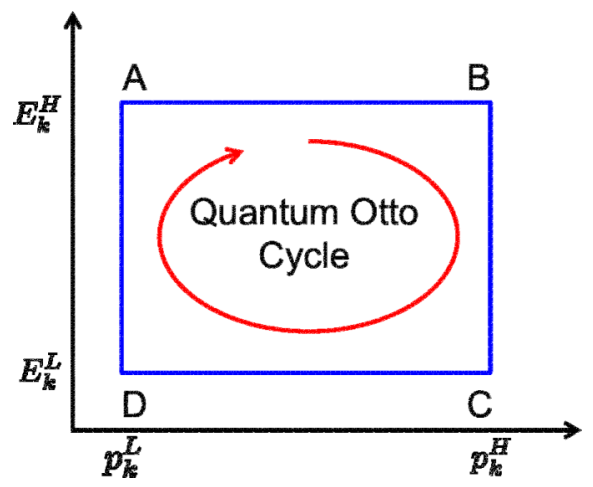

FIG. 1. Quantum Otto engine cycle in the eigenenergy $\left(E_{k}\right)$ and the occupation probability $p_{k}$ space of an arbitrary energy level $k$ of the spin system. In the quantum adiabatic stages, $D \rightarrow A$ and $B \rightarrow C$, the system is disconnected from the heat baths and model parameters $\gamma_{x}, \gamma_{h}$ changes between $\gamma_{x}^{L}, \gamma_{y}^{L}$ and $\gamma_{x}^{H}, \gamma_{y}^{H}$. In the isochoric stages, $A \rightarrow B$ and $C \rightarrow D$, the system is coupled to a hot bath at temperature $T_{H}$ and a cold bath at temperature $T_{L}$, respectively, so that $p_{k}$ changes between $p_{k}^{L}$ and $p_{k}^{H}$.

model from the Landau-Zener perspective, finding significant effects arising from classical rather than quantum interference, the latter being swept out by quantum fluctuations [36]. In the following, we describe the engine cycle and explicitly show how interference contributes to its work output.

\section{B. Quantum Otto engine cycle}

The quantum Otto cycle can be described by an energyprobability $E$ - $p$ diagram depicted in Fig. 1, where $p_{k}$ represents the occupation probability of an arbitrary energy level $k$ of the model Hamiltonian. It is a four stroke irreversible engine that consists of two quantum adiabatic and two quantum isochoric stages [15]. In the adiabatic stages, $D \rightarrow A$ and $B \rightarrow$ $C$, the model Hamiltonian changes by varying the parameters $\gamma_{x, y}$ to $\gamma_{x, y}^{H}$ and $\gamma_{x, y}^{L}$, respectively. In the isochoric stages, the Hamiltonian is fixed but the system is in contact with a hot bath at temperature $T_{H}$ and a cold bath at temperature $T_{L}$, so that each energy level population can change to new values of $p_{k}^{H}$ and $p_{k}^{L}$, respectively. The internal energies at the end of each stage can be calculated as $U_{\alpha}=\operatorname{Tr}\left(\rho_{\alpha} \hat{H}_{\alpha}\right)$, where $\rho_{\alpha}$ and $\hat{H}_{\alpha}$ are the density matrix of the working substance and the Hamiltonian at the point $\alpha=A, B, C$, and $D$, respectively. The relevant thermodynamic quantities of the engine are given by [15]

$$
\begin{aligned}
Q_{\text {in }} & =\sum_{k} E_{k}^{H}\left(P_{k}^{H}-P_{k}^{L}\right), \quad Q_{\text {out }}=\sum_{k} E_{k}^{L}\left(P_{k}^{L}-P_{k}^{H}\right), \\
W & =Q_{\text {in }}+Q_{\text {out }}, \quad \eta=W / Q_{\text {in }},
\end{aligned}
$$

where the heat intake and outtake are denoted by $Q_{\text {in }}$ and $Q_{\text {out }}$, respectively (we use the convention according to which $\left.Q_{\text {out }}<0\right)$.

In a quantum adiabatic process, eigenstates change while the populations remain the same. The multilevel system does not need to be assigned either a real or an effective temperature at points $A$ and $C$, as a nonthermal state is allowed at the end of the quantum adiabatic transformation. In our analysis we do not consider finite time cycles and assume, by the introduction of infinitesimal perturbations, sufficiently slow quantum 
adiabatic transformations can be introduced. In addition we further assume that the target thermal states at points $A$ and $C$ can be achieved at any interaction strengths $\gamma_{x}, \gamma_{y}$ by contact with corresponding reservoirs.

In order to discern the contribution of quantum interference to the work output of the cycle, we apply a perturbative calculation inspired by the density matrix perturbation theory [37], for the calculations of the internal energies $U_{x}$.

\section{Perturbative calculation of internal energies}

We consider $\gamma_{x}^{H}>\gamma_{x}^{L} \gg \gamma_{y}^{L}>\gamma_{y}^{H}>0$. The choice of small values for parameters $\gamma_{y}^{L}, \gamma_{y}^{H}$ allows us to calculate the internal energies perturbatively to reveal the interference physics behind the problem. The ordering of the energies leads to a constructive interference in the work output, as we discuss later on. We consider the terms (cf. Ref. [37] and see Fig. 4 for the regime of validity where the other terms in the expansion are negligible) $U_{\alpha} \approx \operatorname{Tr}\left(\rho_{\alpha}^{(0)} H_{\alpha}^{(0)}\right)+\operatorname{Tr}\left(\rho_{\alpha}^{(0)} H_{\alpha}^{(1)}\right)$ with $\rho_{\alpha}^{(0)}=\sum_{n} P_{n}^{\alpha}|n\rangle\left\langle n\left|, H_{\alpha}^{(0)}=\sum_{n} \gamma_{x}^{\alpha} n^{2}\right| n\right\rangle\langle n|$, and $H_{\alpha}^{(1)}=$ $\sum_{m} \gamma_{y}^{\alpha} m^{2}|m\rangle\langle m|$. Here $|n\rangle$ and $|m\rangle$ are the angular momentum states for the quantization axis $x$ and $y$, respectively, such that $\hat{S}_{x}|n\rangle=n|n\rangle$ and $\hat{S}_{y}|m\rangle=m|m\rangle$ with $n, m=-S$, $-S+1, \ldots, S-1, S$. With this notation

$$
U_{\alpha} \approx \sum_{n} P_{n}^{\alpha} \gamma_{x}^{\alpha} n^{2}+\sum_{n, m} P_{n}^{\alpha} \gamma_{y}^{\alpha} m^{2} P(n, m)
$$

with the transition probabilities $P(n, m)=|\langle n \mid m\rangle|^{2}$ and the occupation probabilities $P_{n}^{\alpha}(\alpha=B, D)$ that are obtained from the canonical distribution

$$
P_{n}^{B}=\frac{e^{-n^{2} \gamma_{x}^{H} / T_{H}}}{\sum_{n} e^{-n^{2} \gamma_{x}^{H} / T_{H}}}, \quad P_{n}^{D}=\frac{e^{-n^{2} \gamma_{x}^{L} / T_{L}}}{\sum_{m} e^{-n^{2} \gamma_{x}^{L} / T_{L}}} .
$$

The output work can thus be written as $W=W_{x}+W_{x y}$, where

$$
W_{x}=\sum_{n}\left(P_{n}^{B}-P_{n}^{D}\right)\left(\gamma_{x}^{H}-\gamma_{x}^{L}\right) n^{2}
$$

and

$$
W_{x y}=\sum_{n, m}\left(P_{n}^{B}-P_{n}^{D}\right)\left(\gamma_{y}^{H}-\gamma_{y}^{L}\right) m^{2} P(n, m) .
$$

The term $W_{x y}$ shows the quantum interference contribution to the work output. It involves the transition probabilities between spin states belonging to different quantization axes, which interfere mutually with weights proportional to the difference in populations, and signs determined by $\gamma_{y}^{H}-\gamma_{y}^{L}$. Such interference can thus be either constructive or destructive, therefore influencing the value of the output work $W$. By taking a low-temperature regime, we can safely assume that $n$ is limited to a few small values (only the low-lying states are populated). We find that the transition probabilities $P(n, \pm S)$ grow as $n$ decreases. Accordingly, the negative population differences associated with the lower levels are weighted more, so that a negative value of the difference $\gamma_{y}^{H}-\gamma_{y}^{L}$ allows for interference that enhances the work output. The effect can be explained geometrically using semiclassical interferences in the spin phase space.

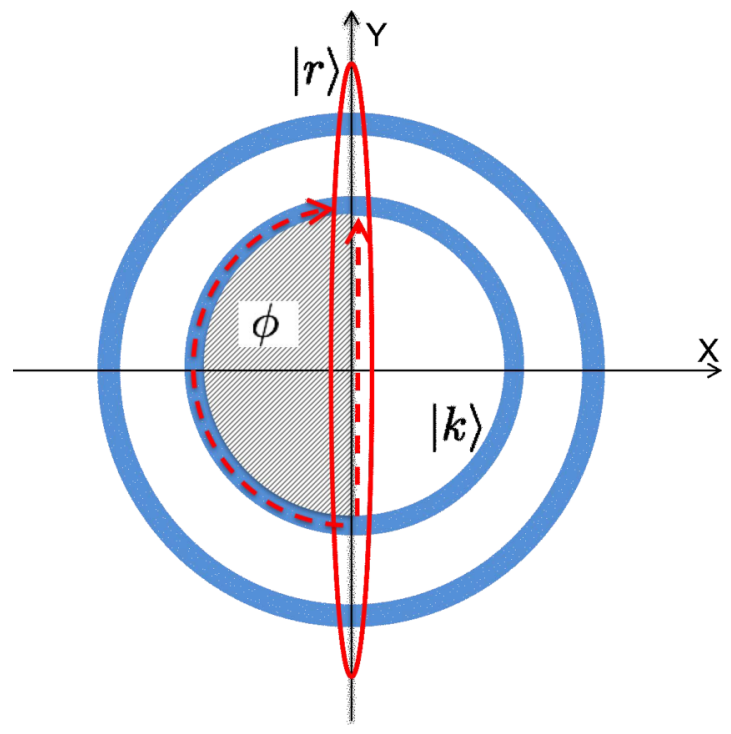

FIG. 2. Interference of a squeezed vacuum state $|r\rangle$ with Fock number states $|k\rangle$ in the oscillator phase space $X, Y$, where $X$ and $Y$ denote the displacement and momentum, respectively. The ellipse and the concentric circular bands represent the squeezed state and the Fock states, respectively. The ellipse cuts two overlap regions, with area $A=A(k, r)$ on the annulus associated with $|k\rangle$. The dashed lines indicate two possible paths connecting the overlap regions. The shaded area between the dashed lines is denoted by $\phi=k \pi$. The interference can be envisioned as a Young interferometer where the overlap regions correspond to the two slits, or a path interference of the trajectories between the overlap regions, or superposition of two waves originating from the overlap regions and propagating in opposite directions. Transition probability between the states is determined by $P(k)=4 A \cos ^{2}(\phi / 2)$.

\section{PHASE-SPACE INTERFERENCE}

The phase-space interference method allows us to use a semiclassical picture to geometrically interpret and visualize quantum interferences. A critical signature of interference is the existence of an oscillatory pattern with respect to some geometrical parameters of the interferometer. In a two-slit Young interferometer, for example, oscillatory behavior emerges with respect to the spatial separation between the slits, translated into the path difference between different arms of the interferometer. In quantum optics, oscillations in the probability distribution of the squeezed vacuum state with respect to the photon number have been explained using a semiclassical interference picture in the oscillator phase space [23].

Fock states, $|k\rangle$, where $k$ is an integer, can be depicted as concentric annuli in the phase space as shown in Fig. 2. Each band has a finite area fixed by the semiclassical BohrSommerfeld quantization condition of the action. Squeezed vacuum state $|r\rangle$, obtained by the squeezing operator, with squeezing parameter $r$, transforming the vacuum Fock state $|0\rangle$, is represented by an ellipse. The probability distribution $P(k)=|\langle k \mid r\rangle|^{2}$ can be visualized as the overlap of the $k$ th circular annulus and the ellipse [24]. The overlap consists of two regions with finite areas, which can be imagined as two slits of a Young interferometer. As the photon number $k$ increases, the overlapping regions get smaller and the distance 
between them increases so that the oscillatory decrease of $P(k)$ with $k$ conforms to the double-slit interferometer behavior. Other interpretations of the interference oscillations can also be considered. First, two signals from one overlap region following two different paths, propagate to the other overlap region. One path is through the circular annulus and the other one is through the ellipse. Signals interfere due to the phase delay between them. Second, superposition of two waves, emitted from the overlap regions in opposite directions, lead to interference [23]. In these interpretations, as the slits gets narrower and the distance between them increases with $k$, we expect an oscillatory decrease of $P(k)$ with $k$, conforming to the analytical calculation showing oscillations in $P(k)$ with respect to even and odd number of photons [23]. The phase delay is determined by the area between the paths [24]. The paths can be approximated by a line and an arc, respectively, for a sufficiently narrow ellipse (large $r$ ). The radius of the circle is $\sim \sqrt{2 k}$, which gives the area between the paths to be $\pi k$. The interference factor due to this phase difference is given by $\exp (i \pi k)=(-1)^{k}$ so that the probability becomes $P(k) \sim 1+(-1)^{k}$.

Bosonic mapping of spin operators by the HolsteinPrimakoff transformation [38] $\hat{S}_{+}=\hat{S}_{-}^{\dagger}=\hat{a}^{\dagger}\left(2 S-\hat{a}^{\dagger} \hat{a}\right)^{1 / 2}$ and $\hat{S}_{z}=\hat{a}^{\dagger} \hat{a}-S$ could allow for using similar phase-space interference methods to investigate quantum interference in spin systems. There is a more direct approach in which it is possible to associate semiclassical interferences in the spin phase space with the quantum interferences [25]. The spin phase space is defined by $\left(S_{x}, S_{y}, S_{z}\right)$ where the spin states can be described on the Bloch sphere of radius $R_{S}=\sqrt{S(S+1)}$ for a given total spin $S$ as shown in Fig. 3 [25]. Each angular momentum state can be represented by a semiclassical Kramers trajectory, similar to the WKB method, the central

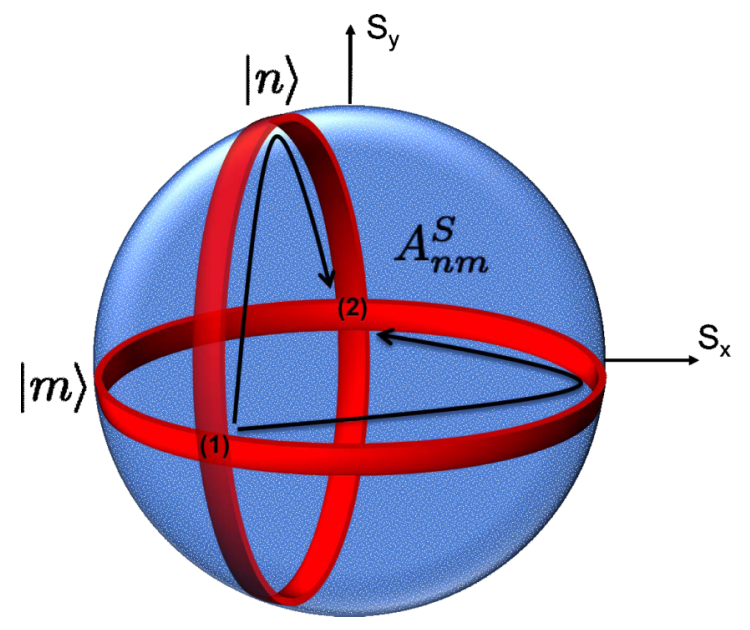

FIG. 3. Interference of a spin state $|n\rangle$ with respect to the quantization axis $x$ and a spin state $|m\rangle$ with respect to the quantization axis $y$. Spin states are represented by circular bands about their corresponding quantization axes. Their overlaps are shown by the symmetric regions (1) and (2), each with area $a_{n m}^{S}$. Among four possible paths from (1) to (2), two paths are shown with solid black curves. The surface area between the indicated paths on the Bloch sphere of radius $R_{S}$ is shown by $A_{n m}^{S}$. The transition probability between the states is determined by $P(n, m ; S)=4\left(a_{n m}^{S} / 2 \pi R_{S}\right) \cos ^{2} \phi_{n m}^{S}$, with $\phi_{n m}^{S}$ being an interference angle determined by $A_{n m}^{S}$ and $R_{S}$. line along a Planck-Bohr-Sommerfeld band on the surface of the Bloch sphere, which is an annulus of width $\sim 1$ about the corresponding quantization axis. For example, the band of state $|n\rangle$ lies between $S_{x}=n+1 / 2$ and $S_{x}=n-1 / 2$ circles for the $x$-quantization axis. Interference, mathematically encompassed into the transition probabilities $P(n, m)$, is geometrically understood as the intersection of two such annuli, one about the $x$-axis and the other about the $y$-axis. There are four paths on the Bloch sphere connecting the intersection points. It has been found that one can choose a particular set of two paths, each on a different circle, and express the transition probability in the form [25]

$$
P(n, m)=4 \frac{a_{n m}^{S}}{2 \pi R_{S}} \cos ^{2}\left(\frac{A_{n m}^{S}}{2 R_{S}}-\frac{\pi}{4}\right),
$$

where $A_{n m}^{S}$ is the area between the paths connecting the overlap points on the Bloch sphere, and $a_{n m}^{S}$ is the area of overlap between the corresponding bands as shown in Fig. 3. The general expressions of these quantities are given in Ref. [25].

\section{QUANTUM INTERFERENCE IN THE ENGINE CYCLE}

According to Eq. (8), semiclassical interference in the spin phase space is associated with the quantum interference of transition probabilities. Due to the additional thermal distribution weight factors in the internal energy expressions, we have a multitude of overlapping Kramers trajectories, which implies the consideration of a thermally weighted phase-space interference grid [39], which might result in a washing out of any oscillatory behavior (a typical signature of interference) in the work output [cf. Eqs. (3) and (6)]. Moreover, care should be used to gauge the nature of the contributions to such oscillations. Indeed, both $W_{x}$ and $W_{x y}$ can exhibit oscillations with integer and half-integer values of $S$, due to the spectral difference of the unperturbed Hamiltonian in $H_{\alpha}^{(0)}$. However, such oscillations would not be related to any quantum interference. In order to discern the quantum interference effects in the work output, we can consider two approaches. One possibility is to measure the general overall work output and its value for $\gamma_{y}=0$, which would give exactly $W_{x}$, and infer $W_{x y}$ from the difference $W-W_{x}$. Alternatively, one can measure the total work output for positive and negative values of the difference $\gamma_{y}^{H}-\gamma_{y}^{L}$, labeling such values as $W_{+}$and $W_{-}$, respectively. The interference term in the work output would then be inferred as $W_{x y}=\left(W_{+}-W_{-}\right) / 2$.

$W_{x}$ is not the significant term for us and we do not demand large changes in the spin-spin interactions in it. We take $\gamma_{x}^{H}=1.01$ and $\gamma_{x}^{L}=1$. Perturbation energies will be smaller and besides we consider constructive interference and take $\gamma_{y}^{H}=0.01$ and $\gamma_{y}^{L}=0.02$. The parameters for the calculation are taken to be dimensionless and scaled with $\gamma_{x}^{L}$. In addition, to make the interference oscillations significant, it is advantageous to limit the number of bands in the interference grid on the Bloch sphere. This can be accomplished by taking $T_{H} \ll \gamma_{x}^{H}$ and $T_{L} \ll \gamma_{x}^{L}$. Temperatures of the heat baths are taken to be $T_{H}=0.4$ and $T_{L}=0.1$ in the same scaling where $\gamma_{x}^{L}=1$.

Before we proceed, a word of caution should be given on the range of validity of the perturbative approach. It works over a finite range of total spin $S$, depending on the parameter 


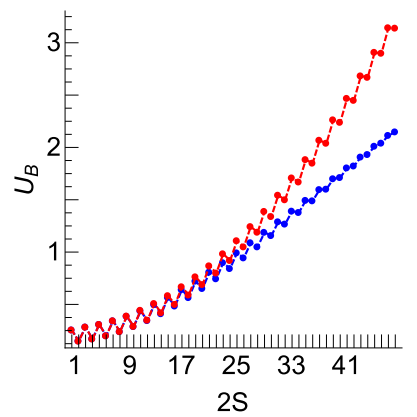

(a)

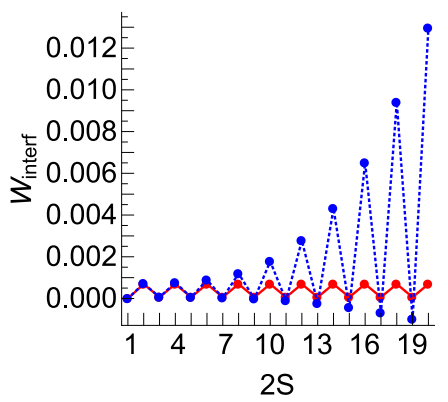

(b)

FIG. 4. Dependence of (a) the internal energy $U_{B}$ at point $B$ and (b) work output of a quantum Otto cycle in Fig. 1 with a working substance of interacting spins on the number of spins $N=2 S$ with total spin $S$. The interaction is described by the LMG model. The upper red curve in (a) and connected red markers (b) are for the perturbative calculation, while the lower blue curve in (a) and the blue marking points in (b) are for the exact diagonalization method. We use dimensionless parameters scaled by $\gamma_{x}^{L}$ and take $\gamma_{x}^{H}=1.01$, $\gamma_{x}^{L}=1, \gamma_{y}^{H}=0.01$, and $\gamma_{y}^{L}=0.02$. The temperatures of the heat baths are $T_{H}=0.4$ and $T_{L}=0.1$.

set used. As far as the internal energies are concerned, the range of $S$ over which the perturbation method can be applied is relatively wide, while perturbative evaluations of work are limited to a relatively smaller range of $S$, as can be seen in Fig. 4. We calculate the work output of the engine $W=Q_{\text {in }}+$ $Q_{\text {out }}$, where $Q_{\text {in }}=U_{B}-U_{A}$ and $Q_{\text {out }}=U_{D}-U_{C}$ by exact numerical diagonalization of the model and the perturbative approach. The changes in $U_{B}$ against the number of spins $N=2 S$ is plotted in Fig. 4(a). A similar behavior is found for the other internal energies at points $A, C$, and $D$, showing oscillations dependent on the parity of $N$. The oscillations are translated to the work output, plotted in Fig. 4(b).

We warn the reader that more correction terms in the density matrix perturbation expansion [Eq. (4)] could be added to bring our results closer to the exact one. However, the calculation of such terms is rather cumbersome, especially for arbitrary temperatures [40]. The terms we keep are based upon a zerotemperature expansion [37]. Yet, they capture the essential physics that is necessary to interpret the exact low-temperature behavior, showing perfect agreement up to small $N(\sim 10)$, with the exact values of the internal energies and the contribution of interference to the work output as shown in Fig. 4.

The growth of $U_{B}$ with $N$ becomes linear in the thermodynamic limit $U_{B} \sim N$ or the internal energy per particle becomes a finite value for large $N$, as shown in Fig. 5(a). We remind one that our analysis is restricted to the maximum total spin sector of the Hilbert space. Consideration of the full Hilbert space leads to the expected nonextensive behavior due to the finite energy left per particle in the thermodynamic limit. The extensive model, applying the Kac's rescaling, gives, however, zero mean energy per particle for large $N$, consistently with the expected thermodynamical limit for an extensive system in the full Hilbert space of $N$ particles.

The work output according to the nonextensive description of the working substance shows a superlinear $\left(\sim N^{2}\right)$ growth with the even number of spins $N$ up to a critical $N_{\max } \sim 30$ and decrease toward zero after this point. The behavior of the even

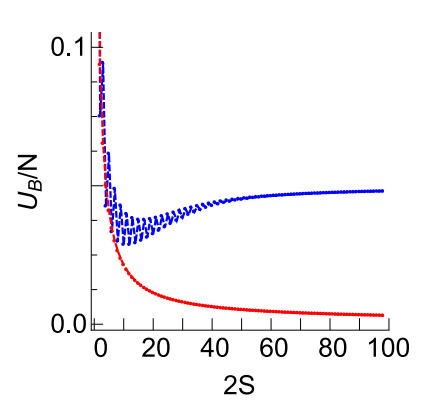

(a)

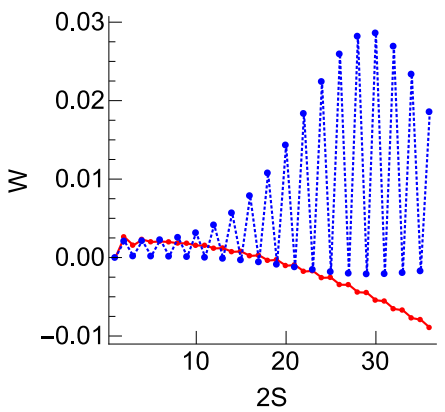

(b)

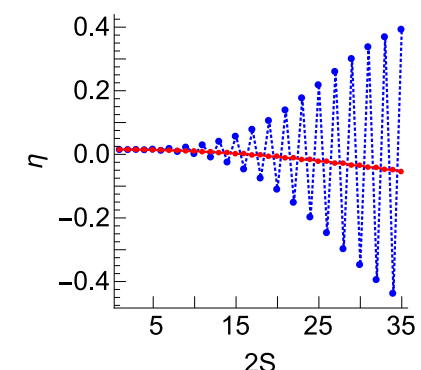

(c)

FIG. 5. Dependence of (a) the internal energy per particle $U_{B} / N$ at point $B$, (b) work output $W$, and efficiency $\eta$ of a quantum Otto cycle in Fig. 1 with a working substance of interacting spins on the number of spins $N=2 S$ with total spin $S$. The interaction is described by the LMG model. The lower red curves in (a) and (b), and the middle red curve in (c) are for the extensive description of the LMG model, while the upper blue curve in (a) and the blue marking points in (b) and (c) are for the nonextensive description. We use dimensionless parameters scaled by $\gamma_{x}^{L}$ and take the spin-spin interaction parameters as $\gamma_{x}^{H}=1.01, \gamma_{x}^{L}=1, \gamma_{y}^{H}=0.01$, and $\gamma_{y}^{L}=0.02$. Temperatures of the heat baths are taken to be $T_{H}=0.4$ and $T_{L}=0.1$.

number of spins is consistent with the microeconomical law of diminishing returns [26] as well as with many-body bosonic QHE [20,21]. In addition to the "point of maximum return," there is a "point of diminishing return" at $N_{\text {dim }} \sim 20$ after which each added spin decreases the rate of work production. However, there is a difference in the microeconomy of quantum labor force according to which a nonextensive working system with an odd number of spins does negligible work or yields even negative returns, while an extensive system can return work. However, even in such latter case, the return is still less than that of the even number of spins [cf. Fig. 5(b)]. In the case of an extensive model, $W$ decreases with $N$, exhibiting oscillations dependent on the parity of the latter. Heat-engine operations with significant $W>0$ are only possible for a small number of spins in an extensive model, and the optimum number of workers is $N=2$. This number coincides with the maximum work at maximum efficiency.

The efficiency is evaluated by $\eta=1+Q_{\text {out }} / Q_{\text {in }}$ and plotted in Fig. 5(c). Both extensive and nonextensive engines have similar efficiencies for small values of $N$. This can be intuitively expected due to the small difference in the control parameters of the adiabatic stages in our set of parameters. The extensive model has negative efficiency after $N \sim 10$ for which the work output is also negative, and the system cannot operate as a heat engine. Efficiency is weakly showing 
even-odd oscillations for both models and small $N$. The point of maximum return $N \sim 30$ does not coincide with the value of $N \gtrsim 35$ at which the maximum efficiency for nonextensive systems is achieved.

The parity-dependent oscillations found in the work and efficiency cannot be immediately associated with the occurrence of quantum interference. However, our perturbative analysis led us to determine a set of parameters for which the oscillations due to spectral differences can be removed from the work output. Accordingly, we conclude that for a sufficiently small number of spins $N<10$ - over which the perturbation approach is justified-parity-dependent oscillations are genuinely due to quantum interference. Exact calculations still predict more significant oscillations for a larger number of spins. However, whether these are due to quantum interference or not cannot be deduced from the first-order density matrix perturbation theory. We leave this as an open problem for future explorations.

\section{ISOLATION OF THE QUANTUM INTERFERENCE}

We have seen that work output of the engine can exhibit even-odd oscillations with respect to the number of quantum workers, which is particularly significant in the case of a nonextensive working system. We can isolate the genuine quantum interference contribution to these oscillations by using either $W_{x y}=W-W_{x}$ or $W_{x y}=\left(W_{+}-W_{-}\right) / 2$ for a sufficiently low number of spins where the perturbation method is satisfactory. Our objective now is to establish a clearer link between semiclassical interference picture in the spin phase space with the even-odd oscillations in the work output. For that aim, let us consider the factors contributing to the work output expression, Eq. (7).

The behavior of the first factor $\Delta P_{n}:=P_{n}^{B}-P_{n}^{D}$, which is the population change of an unperturbed level $n$, is calculated by the Eqs. (4) and plotted in Fig. 6. Other $S$ values give the same result that the dominant $n$ contributing to the work output are $n=0, \pm 1$ and $n= \pm 1 / 2, \pm 3 / 2$ for integer and half-integer $S$. This is enforced by taking $T_{H} \ll \gamma_{x}^{H}$ and $T_{L} \ll \gamma_{x}^{L}$ to restrict the number of the spin bands on the Bloch sphere forming an interference grid (cf. Fig. 3). The relations $\Delta P_{0}=$ $2 \Delta P_{ \pm 1}$ and $\Delta P_{ \pm 1 / 2}=\Delta P_{ \pm 3 / 2}$ hold true for other $S$, too.

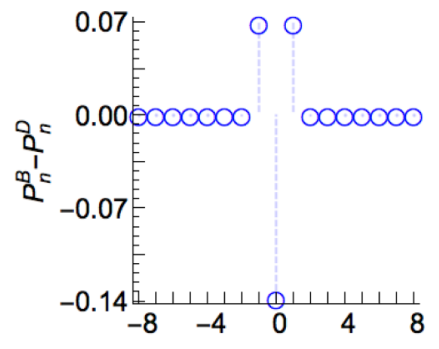

(a) ${ }^{n}$

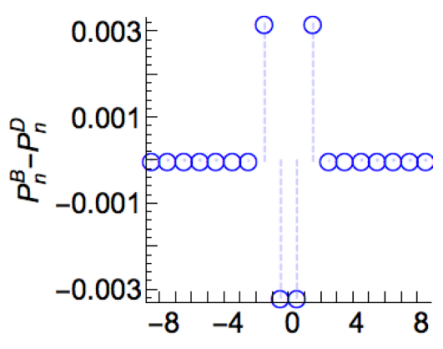

(b) $\mathrm{n}$
FIG. 6. Population change $P_{n}^{B}-P_{n}^{D}$ of unperturbed levels $n$ for (a) the total spin $S=8$ and (b) $S=17 / 2$. Other $S$ values exhibit similar behavior. We use dimensionless parameters scaled by $\gamma_{x}^{L}$ and take the spin-spin interaction parameters as $\gamma_{x}^{H}=1.01, \gamma_{x}^{L}=1$, $\gamma_{y}^{H}=0.01$, and $\gamma_{y}^{L}=0.02$. Temperatures of the heat baths are taken to be $T_{H}=0.4$ and $T_{L}=0.1$.
The second factor is the energy change which increases quadratically with $n$. A spectrum linear in $n$ cannot yield interference oscillations, which can be seen by the symmetry properties of populations and transition probabilities. In contrast to $x$-quantized spin levels $|n\rangle$, which is thermally limited to low levels, energetically higher levels in $y$-quantized spin $|m\rangle$ participate to interference grid. Hence, we can further approximate the dominant bands in the grid by taking $m= \pm S$. As the integer and half-integer $S$ are decoupled in the Hamiltonian, we can deduce that the grid approximately consists of pairs of bands $(n, m)$ which are $(0, \pm S),( \pm 1, \pm S)$ and $\left( \pm 1 / 2, \pm S^{\prime}\right),\left( \pm 3 / 2, \pm S^{\prime}\right)$, for integer $S$ and half-integer $S$, respectively.

The transitions between the intersecting points of these bands lead to interference effects and the work output can be expressed accordingly, as

$$
W_{x y}=4 \Delta P_{k} \Delta \gamma_{y} S^{2}[(P(k, S)-P(k+1, S)],
$$

where $\Delta \gamma_{y}:=\gamma_{y}^{H}-\gamma_{y}^{L}$, and $k=1 / 2$ and $k=0$ for halfinteger and integer $S$, respectively. This formula should only be used for qualitative purposes. While the consideration of $n$ by the few lower bands is a quite good description, the restriction of $m$ by only the highest bands $\pm S$ is a quite poor approximation. In fact $\sum_{m} m^{2} P(n, m)$ differs from $2 S^{2} P(n, S)$ significantly after $S>5$. However, even-odd oscillations and the essential physics are captured qualitatively by limiting ourselves to the $m=S$ band. The other $m$ bands smoothen the oscillatory behavior, making the work output more uniform over even and odd $S$.

We use the semiclassical formula in Eq. (8) for $P(n, m)$ to calculate the work output. The difference between the semiclassical evaluation and the exact value increases toward larger $m$, both for the cases of half-integer [cf. Fig. 7(a)] and integer total spin [cf. Fig. 7(b)]. To improve the agreement, one can use the Airy function fit technique at the tails of $P(n, m)$ [25]. We will not use such methods and be content with a qualitative explanation of the even-odd oscillations from a semiclassical interference picture. The particular qualitative behaviors we look for in the transition probability differences are plotted in Figs. 7(c) and 7(d).

The negativity of the population differences can be associated with the behavior of the interference angle in Eq. (8) which is $\Phi(n, m ; S):=A_{n m}^{S} / 2 R_{S}-\pi / 4$. The largest overlap area is between the states $|n=0\rangle$ and $|m=S\rangle$. It is analytically known that associated $\Phi(0, S ; S)=0$ [25]. Indeed, the surface area of a spherical cap on a sphere of radius $R$ is given by $\pi R h$, with $h$ is the height of the cap. We find $A_{0 S}^{S}=\pi R_{S}\left(R_{S}-S\right)$. For $S \gg 1$ we have $R_{S} \sim S+1 / 2$ so that $A_{S} \sim \pi R_{S} / 2$ which gives $\Phi(0, S ; S) \sim 0$. Other areas between the corresponding bands get smaller and $\Phi \rightarrow-\pi / 4$. These deductions are verified in Figs. 8(a) and 8(b).

We plot $\cos ^{2} \Phi(n, S ; S)$ with respect to $S$ in Fig. 9(a). The differences between the interference cosines for $n=0$ and $n=1 / 2$ are smaller than those for $n=1$ and $n=3 / 2$. The amplitude of the cosines, $\sim 2 / \pi R_{S}$, regularly decrease with $S$ and do not change the hierarchy $P(0, S ; S) \lesssim P\left(1 / 2, S^{\prime} ; S^{\prime}\right)<$ $P(1, S ; S)<P\left(3 / 2, S^{\prime} ; S^{\prime}\right)$ we would deduce by the examination of the interference angles and the cosines, as verified by Fig. 9(b). Here $S$ and $S^{\prime}$ are integer and half-integer total spin values neighboring each other on the real axis. Hence, we 


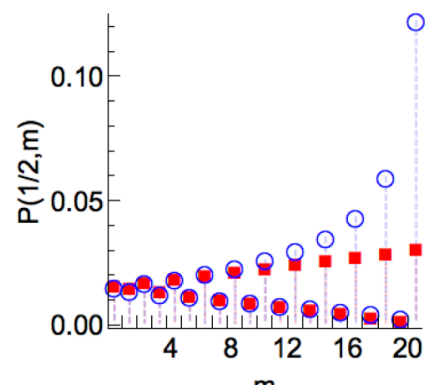

(a)

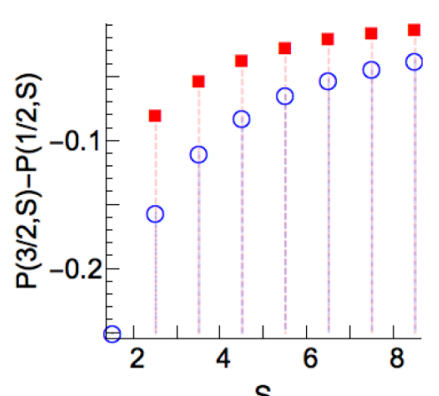

(c) $\mathrm{S}$

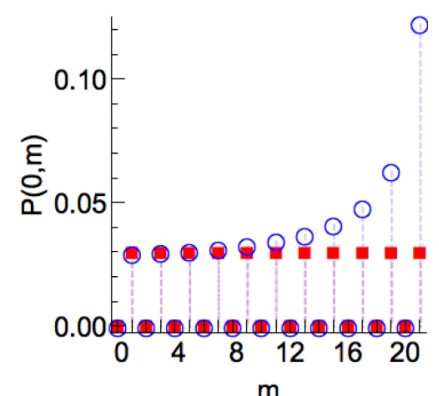

(b)

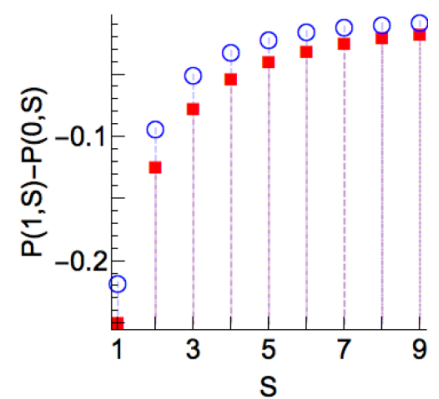

(d)

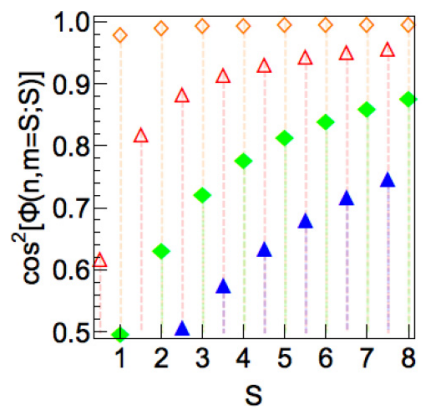

(a)

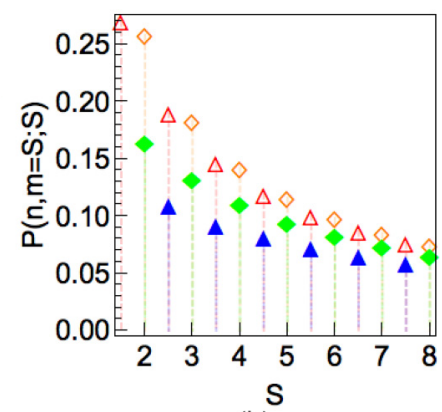

(b)

FIG. 9. Dependence of (a) interference cosine (squared) $\cos ^{2} \Phi(n, m=S ; S)$ and (b) $P(n, m=S ; S)$ on the total spin $S$. Plot markers of empty orange diamond, empty red triangle, filled green diamond, and filled blue triangle are for $n=0, n=1 / 2, n=1$, and $n=3 / 2$, respectively.

those of the odd number of spins. Finally, having seen that $P(k, S)-P(k+1, S)$ is positive, the negativity of the $\Delta P_{k}$ can be compensated by taking the $\Delta \gamma_{y}<0$ so that the interference is tuned to constructive contribution to work output.

\section{CONCLUSION}

We have considered a system of $N$ spins, interacting through extensive and nonextensive LMG-type models, as the working system of a quantum Otto engine. Using the density matrix perturbation method, we have calculated the internal energies, work output, and efficiency of the cycle for both interaction models and compared with the exact solutions. We have found oscillations in the work output with respect to the number of elements of the working medium, more pronounced for the nonextensive model.

We have also provided an intuitive comparison with the microeconomical law of diminishing returns. The work output of a nonextensive engine follows such a law and showcases points of maximum and diminishing returns. The paritydependent oscillations in the output work, however, mark a major difference between quantum and classical working media from a microeconomical perspective.

Our perturbative approach helped us identify the two sources of these oscillations, which are provided by the differences in the Hamiltonian spectrum and the quantum interference between the spin states of different quantization axes contributing to the work output. As the latter contribution can be easily isolated, the fine-tuning of the perturbation parameter can be used to control the (constructive or destructive) character of the interference.

Quantum interference contribution to the work output can be explained by the interplay of thermodynamics, interference in the spin phase space, and nonlinearity of the spin-spin interaction. This suggests that the parity-dependent oscillations in the interference term of the work output associated with the number of quantum workers is a genuine quantum thermodynamical effect.

Our results elevate quantum heat engines to the role of fruitful platforms for the fundamental study of quantum interference. Moreover, the identification of points of maximum and diminishing returns, as quantum analog of the classical law 
of diminishing returns, can be used to optimize the preparation of extensive and nonextensive working substances of quantum heat engines.

\section{ACKNOWLEDGMENTS}

A.Ü.C.H. acknowledges support from the Villum Foundation through a postdoctoral block stipend. Ö.E.M. and
A.Ü.C.H. acknowledge the support by the University Research Agreement between Lockheed Martin Chief Scientist's Office and Koç University. M.P. acknowledges support from the DfESFI Investigator Programme (Grant No. 15/IA/2864). Ö.E.M. and M.P. are grateful to the Royal Society for support through the Newton Mobility Grant scheme (Grant No. NI160057). Ö.E.M. acknowledges support by TUBITAK (Project No. 116F303) and EU COST Action CA15220.
[1] S. Abe et al. (The KamLAND Collaboration), Precision Measurement of Neutrino Oscillation Parameters with KamLAND, Phys. Rev. Lett. 100, 221803 (2008).

[2] S. A. Werner, R. Colella, A. W. Overhauser, and C. F. Eagen, Observation of the Phase Shift of a Neutron Due to Precession in a Magnetic Field, Phys. Rev. Lett. 35, 1053 (1975).

[3] R. Colella, A. W. Overhauser, and S. A. Werner, Observation of Gravitationally Induced Quantum Interference, Phys. Rev. Lett. 34, 1472 (1975).

[4] W. Ehrenberg and R. E. Siday, The refractive index in electron optics and the principles of dynamics, Proc. Phys. Soc., London, Sect. B 62, 8 (1949).

[5] Y. Aharonov and D. Bohm, Significance of electromagnetic potentials in the quantum theory, Phys. Rev. 115, 485 (1959).

[6] R. P. Feynman and A. R. Hibbs, in Quantum Mechanics and Path Integrals, emended edition, edited by D. F. Styer (Dover, Mineola, NY, 2010).

[7] H. J. Lipkin, N. Meshkov, and A. J. Glick, Validity of many-body approximation methods for a solvable model, Nucl. Phys. 62, 188 (1965).

[8] R. G. Unanyan and M. Fleischhauer, Decoherence-Free Generation of Many-Particle Entanglement by Adiabatic Ground-State Transitions, Phys. Rev. Lett. 90, 133601 (2003).

[9] C. Lee, Adiabatic Mach-Zehnder Interferometry on a Quantized Bose-Josephson Junction, Phys. Rev. Lett. 97, 150402 (2006).

[10] A. Micheli, D. Jaksch, J. I. Cirac, and P. Zoller, Many-particle entanglement in two-component Bose-Einstein condensates, Phys. Rev. A 67, 013607 (2003).

[11] G. J. Milburn, J. Corney, E. M. Wright, and D. F. Walls, Quantum dynamics of an atomic Bose-Einstein condensate in a doublewell potential, Phys. Rev. A 55, 4318 (1997).

[12] M. Kitagawa and M. Ueda, Squeezed spin states, Phys. Rev. A 47, 5138 (1993).

[13] S. Morrison and A. S. Parkins, Dynamical Quantum Phase Transitions in the Dissipative Lipkin-Meshkov-Glick Model with Proposed Realization in Optical Cavity QED, Phys. Rev. Lett. 100, 040403 (2008).

[14] A. Campa, T. Dauxois, and S. Ruffo, Statistical mechanics and dynamics of solvable models with long-range interactions, Phys. Rep. 480, 57 (2009).

[15] H. T. Quan, Y.-x. Liu, C. P. Sun, and F. Nori, Quantum thermodynamic cycles and quantum heat engines, Phys. Rev. E 76, 031105 (2007).

[16] R. Kosloff and A. Levy, Quantum Heat Engines and Refrigerators: Continuous Devices, Annu. Rev. Phys. Chem. 65, 365 (2014).

[17] N. Lambert, Y.-N. Chen, Y.-C. Cheng, C.-M. Li, G.-Y. Chen, and F. Nori, Quantum biology, Nat. Phys. 9, 10 (2013).
[18] S. F. Huelga and M. B. Plenio, Vibrations, quanta and biology, Contemp. Phys. 54, 181 (2013).

[19] R. Kosloff, Quantum thermodynamics: A dynamical viewpoint, Entropy 15, 2100 (2013).

[20] J. Jaramillo, M. Beau, and A. del Campo, Quantum supremacy of many-particle thermal machines, New J. Phys. 18, 075019 (2016).

[21] M. Beau, J. Jaramillo, and A. del Campo, Scaling-up quantum heat engines efficiently via shortcuts to adiabaticity, Entropy $\mathbf{1 8}$, 168 (2016).

[22] N. Killoran, S. F. Huelga, and M. B. Plenio, Enhancing lightharvesting power with coherent vibrational interactions: A quantum heat engine picture, J. Chem. Phys. 143, 155102 (2015).

[23] W. Schleich and J. A. Wheeler, Oscillations in photon distribution of squeezed states and interference in phase space, Nature (London) 326, 574 (1987).

[24] W. Schleich, D. F. Walls, and J. A. Wheeler, Area of overlap and interference in phase space versus Wigner pseudoprobabilities, Phys. Rev. A 38, 1177 (1988).

[25] C. C. Lassig and G. J. Milburn, Interference in a spherical phase space and asymptotic behavior of the rotation matrices, Phys. Rev. A 48, 1854 (1993).

[26] D. McTaggart, C. Findlay, and M. Parkin, Microeconomics, 4th ed. (Addison Wesley, Frenchs Forest, NSW, 2003).

[27] A. Ü.C. Hardal and Ö. E. Müstecaplioğlu, Superradiant quantum heat engine, Sci. Rep. 5, 12953 (2015).

[28] D. Türkpençe, F. Altintas, M. Paternostro, and Ö. E. Müstecaplioğlu, A photonic Carnot engine powered by a spin-star network, Europhys. Lett. 117, 50002 (2017).

[29] R. Uzdin, A. Levy, and R. Kosloff, Equivalence of Quantum Heat Machines, and Quantum-Thermodynamic Signatures, Phys. Rev. X 5, 031044 (2015).

[30] J. Larson, Circuit QED scheme for the realization of the LipkinMeshkov-Glick model, Europhys. Lett. 90, 54001 (2010).

[31] J. Campos and J. Hirsch, Single molecule magnets and the Lipkin-Meshkov-Glick model, Rev. Mex. Fis. S 57, 56 (2011).

[32] T. Opatrný, M. Koláŕ, and K. K. Das, Spin squeezing by tensor twisting and Lipkin-Meshkov-Glick dynamics in a toroidal Bose-Einstein condensate with spatially modulated nonlinearity, Phys. Rev. A 91, 053612 (2015).

[33] M. Kac, G. E. Uhlenbeck, and P. C. Hemmer, On the van der Waals theory of the vapor-liquid equilibrium. I. Discussion of a one-dimensional model, J. Math. Phys. 4, 216 (1963).

[34] C. Tsallis, Possible generalization of Boltzmann-Gibbs statistics, J. Stat. Phys. 52, 479 (1988).

[35] M. Campisi and R. Fazio, The power of a critical heat engine, Nat. Commun. 7, 11895 (2016).

[36] J. Larson, Interaction-induced Landau-Zener transitions, Europhys. Lett. 107, 30007 (2014). 
[37] A. M. N. Niklasson and M. Challacombe, Density Matrix Perturbation Theory, Phys. Rev. Lett. 92, 193001 (2004).

[38] T. Holstein and H. Primakoff, Field dependence of the intrinsic domain magnetization of a ferromagnet, Phys. Rev. 58, 1098 (1940).
[39] M. J. Gagen, Phase-space-interference approaches to quantum superposition states, Phys. Rev. A 51, 2715 (1995).

[40] A. M. N. Niklasson, M. J. Cawkwell, E. H. Rubensson, and E. Rudberg, Canonical density matrix perturbation theory, Phys. Rev. E 92, 063301 (2015). 